\title{
Quaderni
}

QUADERNI Communication, technologies, pouvoir

86 | Hiver 2014-2015

Penser la politique par le film

\section{Temporalité de la politique alternative dans les} séries

Antoine Faure et Emmanuel Taïeb

\section{OpenEdition}

Édition électronique

URL : http://journals.openedition.org/quaderni/861

DOI : 10.4000/quaderni.861

ISSN : 2105-2956

Éditeur

Les éditions de la Maison des sciences de l'Homme

Édition imprimée

Date de publication : 5 janvier 2015

Pagination : 23-37

Référence électronique

Antoine Faure et Emmanuel Taïeb, «Temporalité de la politique alternative dans les séries », Quaderni [En ligne], 86 | Hiver 2014-2015, mis en ligne le 05 janvier 2017, consulté le 02 mai 2019. URL : http:// journals.openedition.org/quaderni/861; DOI : 10.4000/quaderni.861 


\section{$D$ ossier}

\section{Temporalité de la politique alternative \\ dans les séries}

Antoine

Faure

Sciences Po Grenoble

PACTE

Emmanuel

Taïeb

Institut Universitaire de France

Sciences Po Lyon

Triangle
La multiplication des séries dans la programmation des chaînes de télévision et leur succès critique attise la curiosité des universitaires. Les séries ont fait l'objet de colloques et de séminaires ${ }^{1}$, ou même de thèses de doctorat ${ }^{2}$, et diverses publications sont venues alimenter la cumulativité et la réflexivité de ces analyses, soit à partir de monographies, soit en interrogeant le statut de l'objet série et les manières de le saisir depuis les sciences sociales. Ce phénomène est relativement nouveau : longtemps les séries sont restées au purgatoire de la critique, comme un sous-genre appartenant à l'univers jugé illégitime de la télévision. Ce snobisme avait été dénoncé en d'autres temps. Par Serge Daney, par exemple, qui, en affirmant qu'il y avait " séries » et « séries », entendait rappeler l'inventivité narrative et la qualité de certaines séries classiques comme Columbo $^{3}$. Aussi la volonté de Daney d'être «passeur » de ce genre méprisé apparaît d'autant plus courageuse qu'il écrit à une période qui, rétrospectivement, apparaît comme une " préhistoire » des séries, tant au niveau des modes de production, des thèmes, que des modes de narration. Cet élitisme n'a cependant pas totalement disparu aujourd'hui, et une série comme The Wire, considérée comme incontestablement légitime, fait parfois l'objet d'un investissement exclusif de certains chercheurs, tandis que nombre d'autres séries ne sont ni regardées ni pensées par les mêmes.

Or, il nous semble que The Wire est surtout représentative d'un saut qualitatif plus général, caractérisé par une autonomisation de l'objet narratif « série » des standards existants à la télévision ${ }^{4}$, sans nécessairement se rapprocher de la narration cinématographique. Selon nous, 
l'acte fondateur de cette entrée des séries dans la « maturité » sérielle peut être trouvé dans la diffusion des trente épisodes de Twin Peaks sur ABC en 1990-1991. Plusieurs caractéristiques permettent d'y voir une évolution structurante : un showrunner, véritable " auteur » de la série (deux ici en fait, David Lynch et Mark Frost) ; la lenteur assumée de la temporalité de l'action; une sérialité régulière mais peu soutenue (les épisodes sont longs et d'une fréquence hebdomadaire ou bimensuelle); des personnages évoluant avec le temps, gardant la mémoire des actions passées (mémoire diégétique), et s'y référant continuellement; et une intrigue qui ne se clôt pas réellement, toujours déceptive, laissant des « pierres d'attente » narratives, comme autant de frustration et de désir de reprise chez le spectateur $^{5}$ (on pense aussi au dernier épisode de Lost ou des Soprano). La rupture introduite réconcilie les formes sérielles et le récit, à la différence des soap ou feuilletons qui jouent avant tout sur la répétition comme reproductibilité de schémas narratifs figés et identiques. Ces productions se distinguent donc dans l'histoire des séries : il ne s'agit plus en effet d'un matériau répétitif, de séquences courtes qui «favorisent un perpétuel surgissement de données nouvelles dans la narration ${ }^{6} »$. Chaque épisode n'est plus l'imitation scénaristique des précédents, comme c'était le cas de Columbo ou de Dallas : l'intrigue s'approfondit et monte en puissance au fil des épisodes, en tendant vers une « fin » qui replace les enjeux fictionnels dans un temps long en relation permanente avec le réel.

C'est précisément cette temporalité particulière des séries contemporaines que nous voudrions interroger. Par « séries contemporaines », nous entendons les séries à caractère dramatique et fictionnel, ce que les Américains appellent «drama » (hors sitcom et soap, donc), produites et diffusées depuis une quinzaine d'années, en particulier, mais pas seulement les shows diffusés par la chaîne HBO aux États-Unis ${ }^{7}$. Il nous semble que l'une des caractéristiques essentielles, et souvent négligée, de ces séries est le jeu qu'elles introduisent avec la temporalité comme support d'une réflexion sur le temps collectif. Notre hypothèse est que le temps mis en scène dans les séries est le temps d'une politique alternative. Même dans des séries réalistes ou à vocation documentaire, le temps représenté est un temps « autre », un temps de l'expérimentation, de l'utopie réaliste, de la subversion, de l'explicitation, ou éventuellement de la reconduite de l'ordre social. Sous cet aspect, il s'avère que le temps du monde réel est le passé du temps de la série ; un moment évident et dont les références sont partagées, mais dont on s'éloigne pour construire la fiction. Le temps présent de la série sera, lui, le lieu des anticipations et des propositions, capables de fédérer une communauté de spectateurs. Pour illustrer cette hypothèse, une série comme True Blood commence sa narration en s'appuyant sur un passé qui reconduit des conventions connues (par convention, il existe des vampires ; et il n'est pas besoin de rappeler les mythologies qui s'attachent à cette figure), et que son présent met en scène ce qui se passe à partir du moment où les vampires ont révélé leur existence aux mortels. De cette manière, les séries sont « toujours-déjà » dans un temps fictionnel, plus ou moins plausible, qui est le ressort du déploiement de questions sur le devenir collectif, sur ce que pourrait être littéralement un autre temps partagé socialement et politiquement, qui propose de refonder la communauté. C'est ici 
que s'articule cette temporalité particulière aux drama et le caractère alternatif de la politique qu'ils proposent : c'est par un rapport particulier au temps que la communauté est " réformée » dans les séries. La dilatation de la narration de nombre de séries sert alors à explorer dans tous ses aspects cette autre temporalité ; et au passage c'est aussi ce qui peut contribuer à expliquer le succès de ces programmes (même si l'on ne peut que rester au stade de l'hypothèse sur ce dernier point). C'est donc notamment dans leur temporalité propre, et sa relation au temps social, que les séries font preuve d'un caractère politique, celui de « la » politique de Jacques Rancière : la sortie de l'identification, l'émancipation, la subjectivation, la re-fondation de la communauté, dont elles sont le réceptacle toujours renouvelé ${ }^{8}$.

Les séries seront ici interrogées à partir de l'idée que leur matériau filmique singulier (procédés narratifs, découpage, montage, traitement de l'image, styles scénaristiques, rythme et durée, construction des personnages, utilisation de la musique, etc.) est tout entier mis au service du renouvellement des formes de la temporalité fictionnelle. Comment, à partir de leurs temporalités propres, les séries télévisées contemporaines contribuent-elles à discuter l'ordre politique, économique et social des sociétés occidentales, à le subvertir, et à dévoiler les pistes de son remplacement ? Dans quelle mesure le façonnage temporel des modalités d'appréhension du réel dans les séries permet-il le déploiement d'un nouveau rapport politique au monde?

Trois éléments de réponse nous semblent pouvoir être indiqués. Le premier s'arrêtera sur la grammaire narrative propre aux séries, qui leur permet justement de forger leur propre temps politique. Le deuxième s'arrêtera sur les formes prises par ces présents fictionnels caractéristiques des séries. Le dernier élément interrogera les communautés et mondes alternatifs mis en scène par ces narrations, leur « politique », à partir de leurs temporalités données.

\section{La mise en série : une grammaire narrative du sensible}

La caractéristique fondamentale des séries télévisées est inscrite de façon performative dans le nom de ce format télévisuel : elles ne reflètent ni ne répètent le réel, elles mettent en série des représentations du monde. Le contrat de signification qui leur est propre - soit les interactions entre le showrunner et les producteurs, l'interprétation par les réalisateurs et les acteurs, la programmation par les chaînes de télévision, et la réception par le public -, se définit avant tout à partir des opérations de découpage, de montage et de tressage de l'intrigue en plusieurs épisodes et saisons, donc à partir de la perception de l'agencement sériel des images-mouvements ${ }^{9}$ sur une longue durée. En ce sens, les lectures possibles des séries contemporaines, notamment leur lecture sensible, ne rendent pas seulement compte d'une vision de la réalité, mais incorporent aussi une grammaire narrative. Cette grammaire signale que les séries sont des canaux de représentation des phénomènes sociaux dans un temps qui leur est propre.

Ce système énonciatif est d'une part donné dès le générique, construit en collage d'images, jusqu'au télescopage. Il conserve sa fonction classique de «fiche d'identité » de la série, ou de restitution de son atmosphère (la prégnance du 
vaudou dans True Blood, celle de la mort dans Six Feet Under), mais il annonce également la place centrale du temps. Le générique des Soprano est ainsi celui du parcours chaque jour répété du protagoniste, celui de Six Feet Under, fait de mouvements de caméra et de fondus enchaînés, évoque l'engloutissement par le temps des êtres vivants. À l'inverse, le générique de Game of Thrones ne met en scène que la géographie où se déroulera l'action. D'autre part, le découpage en épisodes et en saisons noue un dense trafic d'images, dans un récit jamais vraiment fini. Cet « aboutissement inachevé » s'observe bien dans une série comme The Wire. Malgré les changements de focales et la complexification du monde dans l'articulation des différents enjeux liés à la ville de Baltimore (délinquance, survie économique des secteurs industriels sinistrés, conquête électorale du pouvoir politique, système éducatif, et traitement journalistique), et une conclusion en forme de funérailles, l'éternel recommencement de l'économie souterraine apparaît sous les traits de Marlo Stanfield, nouveau boss local, qui fait encore monter la violence d'un cran. Avec la fausse oraison funèbre offerte par le corps policier à l'enquêteur Jimmy McNulty dans la dernière scène, le récit se clôt mais les logiques politiques, économiques et sociales décrites persistent et persisteront. Il en va de même dans Six Feet Under, où dans une réponse circulaire à la scène d'ouverture (marquée par la mort du fondateur de la lignée), tous les membres de la famille Fischer et leurs proches sont liquidés un à un (disparitions présentées sous la même forme que les morts initiales de chaque épisode), sans pour autant que les méandres psychologisants de leur quotidien ne soient suturés. En quelque sorte, personnages et mondes de la série lui survivront.
Par ce nouveau « contrat de visibilité » qui fonde les séries contemporaines, le temps qu'elles développent est un temps qui privilégie la cumulativité du récit. La réitération permanente des formats trouve sa fonction dans l'accumulation et l'évolution des éléments narratifs, et pas simplement dans la succession prévisible des épisodes. Si les séries des années 1980, comme Dallas, entendaient jouer sur la durée par la multiplication du nombre d'épisodes, les séries contemporaines se caractérisent par l'incorporation d'une temporalité proche de la temporalité réelle dans le temps même de la série, et parfois avec un nombre d'épisodes assez réduit.

On a ainsi pu saluer le tour de force ou la nouveauté que constituait une série « en temps réel » (avec des aménagements, cependant) comme 24 chrono, ou bien encore une enquête bouclée durant le temps de la saison (The Wire, The Killing), mais on s'est moins interrogé sur la nécessité qui présidait à ce contrat de visibilité. Or, précisément, ce qui se joue dans ce type de séries, c'est l'idée qu'il faut rendre compte de l'inscription des personnages dans le temps biologique général, et que la meilleure façon de réussir cette inscription est de les faire aller à la « vitesse » normale, c'est-à-dire celle du spectateur, celle du temps courant. L'objectif ici est sans doute double : d'un côté, proposer une temporalité de l'action qui soit familière, reconnaissable, linéaire, sans ellipse marquée ; de l'autre montrer l'expérience du temps des personnages (et des acteurs). Dès lors, l'impression de lenteur ou de temps dilaté éprouvée à la vision de certaines séries - les premiers épisodes de la saison 1 de The Wire étant souvent mentionnés comme illustration -, est une impression 
trompeuse, car le temps filmé se confond en fait avec le temps réel, sans aller moins vite -, et à moins d'imaginer une série tout entière au ralenti, comment le pourrait-il ? $^{10}$

Un tel procédé n'est jamais absolu puisque les formes sérielles s'articulent aussi autour de la répétition, des variations en boucle et des discontinuités. Mais ce procédé peut concerner la série entière ou bien seulement certaines scènes, qui fonctionnent alors comme des « trouées » de temps réel dans la narration globale, des moments de « respiration » où justement le changement de rythme est perceptible. C'est le cas par exemple des moments où Tony Soprano est chez sa psychiatre. La plupart du temps filmés en champ-contre-champ, dans un espace clos soustrait à l'agitation extérieure, avec un plan général montrant les deux protagonistes face-àface au début, ces moments de cure apparaissent comme des périodes arrachées à la trame du récit, comme des moments où la parole voit son statut modifié (si Tony Soprano est habituellement directif, il subit ici une maïeutique), et où le récit, devenu brutalement « annexe », fait l'objet d'un bilan ou d'une mise en perspective. On observe le même type de trouées dans la narration lors des scènes de beuverie et de chansons avinées dans The Wire, ou avec les monologues de Rusty dans True Detective. Si pour David Buxton, ces respirations sont des « ralentisseurs » qui posent un problème formel en ce qu'ils ne serviraient qu'à remplir le temps entre deux « séquences motrices $»^{11}$, on peut aussi les lire classiquement comme des moyens de faire découvrir d'autres facettes des personnages, mais aussi comme des moments volés et suspendus dans le récit le plus vaste. La marque des séries contemporaines se lit précisément dans leur capacité à se préoccuper autant des moments forts que des moments qui le sont moins, et d'utiliser ces moments pour pousser l'exploration des protagonistes et l'intrigue dans de nouvelles directions.

La façon dont ce temps réel est occupé varie cependant d'une série à l'autre. Il est saturé d'action dans 24 chrono, au point de lui conférer une totale artificialité, car ce que fait le héros de son temps relève davantage de l'ubiquité vertigineuse que du réalisme. Tandis qu'il peut être plus distendu ailleurs. On pense par exemple à cette séquence de la première saison de Breaking Bad, où le personnage principal tient prisonnier un dealer ennemi dans la cave de son appartement, et traverse mille affres, longuement filmées, pour savoir quel sort lui réserver. Mais l'on songera aussi aux planques et aux écoutes de bandes dans The Wire, ou bien encore à la temporalité fragile des protagonistes de Six Feet Under, toujours surplombée par la mort qui rôde.

Cette expérience du temps joue non seulement sur l'aspect documentaire souvent noté de ces séries, mais surtout fonde la cumulativité expérientielle des protagonistes. L'une des différences les plus marquantes entre les séries plus anciennes et les séries contemporaines est bien que, dans les premières, les protagonistes recommencent chaque histoire comme si toute mémoire des épisodes précédents avait été oblitérée, dans un présent absolu, tandis que la force des séries actuelles est précisément d'insister sur le fait que tout ce qui s'est déroulé jusqu'à présent s'imprime dans l'esprit des personnages et détermine l'ensemble de leurs actions. Le temps se dépose en eux, et fait son œuvre. Ce que les 
personnages ont vécu et ce qu'ils savent est connu d'eux et des spectateurs qui ont suivi l'histoire, sans que l'un ou l'autre aille plus vite. Cette temporalité partagée permet alors aux scénaristes de la tenir pour acquise, comme postulat du récit, et de pouvoir développer l'innovation continue dans des présents alternatifs, par la disposition en images, en mouvement et en série, de modèles de récits, de fictions et de variations du discours ${ }^{12}$.

\section{Présents au pluriel : la politique de la vrai- semblance}

Dans les séries télévisées, le politique est constitué comme un temps primordial qui administre le souvenir de la société réelle. La tension entre l'imaginaire sériel et la réalisation de celui-ci dans une mémoire de l'ordre du monde joue alors sur la vraisemblance des narrations (et de leur mise en forme). À partir de ce passé commun, les séries télévisées proposent des lignes de fuite thématiques dont l'objectif est de mettre en scène une pluralité de présents anticipés. La politique des séries se joue alors dans l'écart entre la mémoire de la communauté du passé et ses alternatives dans le présent des séries.

\section{Une société scientiste enchantée}

L'espace manque ici pour faire une cartographie complète des présents alternatifs proposés par les séries, qui sont fondamentalement ce qui les fait exister. Parmi ces lignes de fuite, encore peu analysées, se trouve le triomphe d'une société scientiste, ou, pour être plus précis, le triomphe de la science et de la technologie dans des sociétés qui paraissent en avoir oublié les vertus et la puissance. Clairement, l'idée que, face au religieux ou à l'obscurantisme, la science est un excellent moyen de médiatiser le rapport au monde social, et de le comprendre, est à la base d'un nombre important de séries : Les Experts, bien sûr (série originale et spin-offs), qui fonctionnent sur le « paradigme de la trace $»^{13}$, et entendent résoudre les crimes en analysant scientifiquement les indices et les signes laissés par le meurtrier. Comme Urgences en son temps, la série s'enorgueillit de ne mettre en scène que des outils existant réellement, mais sa dimension fictionnelle tient dans la réunion en un même lieu de tous ces instruments habituellement dispersés. Le temps long des expériences de laboratoire est, en outre, accéléré, avec une mise en scène obéissant toujours au même schéma : une séquence très découpée pour signifier à la fois l'ellipse temporelle et la densité d'actions réalisées et une musique d'ambiance ou triphop qui entend marquer l'évidence de ce qui se joue avec les instruments scientifiques pour les protagonistes dans cette néo-société scientiste. Dans Numbers, un agent du FBI est aidé par son frère, mathématicien de génie, qui modélise les modes d'action des criminels et permet de les arrêter. De façon plus marginale, la science est un adjuvant de séries comme Nip/Tuck, où le savoir médical est maintenant capable de toutes les prouesses, et où plusieurs épisodes jouent d'ailleurs clairement du côté de l'anticipation (greffe d'une oreille humaine cultivée sur une souris). Dans True Blood, c'est l'apparition, préalable au récit, d'un sang artificiel évitant aux vampires de boire le liquide vital des humains, qui les conduit à sortir de leur situation d'êtres clandestins, et ouvre le développement de l'univers de fiction où les vampires investissent la cité. 
Pour autant, ces séries reposant sur le triomphe de la science suspendent le réel à leur manière. Le temps de l'action s'y déroule le plus souvent dans un lieu confiné (laboratoire, hôpital, scène de crime transformée en espace de déploiement de la science), figurant un monde absent, compris uniquement comme déclencheur d'occasions tragiques nécessitant un recours à la science (le crime rôde, la maladie frappe). On peut aussi se rappeler l'épisode à huis-clos de Breaking Bad, où les deux «cuisiniers » chassent une mouche qui menace de contaminer la pureté de leur solution. L'univers scientifique des séries repose aussi sur l'archivage vertigineux et totalitaire de tous les pans du monde social. C'est ainsi que, dans Les Experts, il est implicitement admis que les agents accèdent, pour les besoins de l'enquête, à d'immenses et improbables bases de données recensant tous les objets imaginables (empreintes digitales, bien sûr, mais aussi formes de pneu, modèles de voitures, de plantes, d'animaux, etc.). Comme l'utopie étrange d'une société orwellienne souriante, où la criminalité serait une pure affaire d'algorithmes. La grammaire narrative de cette série montre bien ces choix d'appropriation du réel à travers le temps de la série : une fois l'enquête débutée, tout se noue sur le temps des manipulations laborantines. Les dialogues se cantonnant alors dans des couloirs et des lieux de passage où les personnages discutent des résultats des analyses. La rapidité des scènes de lecture interprétative des indices examinés montre bien qu'elle est la seule qui puisse être proposée et acceptée.

La mise en série du réel est alors précisément ce qui le met à distance, car ce que les épisodes donnent à voir relève alors plutôt de l'anticipa- tion. De la même façon, si les instruments utilisés existent bien, leur concentration en un même lieu, le temps pris pour les faire parler et faire parler les éléments de preuve, jusqu'au plus petit microobjet, relève d'une temporalité longue, celle de l'expérience scientifique, improbable en-dehors du monde académique. Donc, paradoxalement, même si la science se veut libératrice de l'opacité du monde social, elle apparaît comme une instance définitive et incontournable, qui conteste moins l'ordre social qu'elle ne le reconduit dans une version enchantée de la société technicienne.

\section{Trois icônes du présent sériel}

Malgré la diversité des thématiques et des intrigues proposées, le récit des séries télévisées dessine régulièrement le temps historique de l'appartenance à la communauté, à partir des représentations renouvelées de l'homo æeconomicus, de «l'inadapté » et des forces de l'ordre. La particularité de ces figures inattendues (sauf sans doute pour la police, mais elle est d'un nouveau type) est qu'elles indiquent à quel point le présent proposé reste dans un état fluctuant entre l'anodin et l'imparfait, le quotidien et le brutal, le précaire et le discontinu. Refaire communauté ne se fait pas dans une quelconque évidence, et la fiction permet de proposer de nouvelles icônes qui ne peuvent être construites que dans la grammaire narrative et les temporalités caractéristiques des séries actuelles. À partir de ces figures, les séries entendent définir un autre rapport pratique de l'homme au monde, sorte d'aphorisme imagé qui se fait le langage du monde dans le présent.

La figure traditionnelle de l'homo oeconomicus et sa capacité entrepreneuriale sont ici mises au 
service du trafic de drogue et plus généralement de l'économie souterraine, de façon originale. Par exemple, dans Breaking Bad, le personnage énigmatique de Skyler White prend peu à peu part au trafic de méta-amphétamines de son mari en s'appuyant sur ses compétences de comptable. Dans le déploiement du temps de la série, l'enjeu moral de la revente de narcotiques perd son sens face aux exigences de rigueur financière et économique introduite par son expertise. Au fil des saisons de The Wire, les deux « B » (Stringer Bell et Avon Barksdale) s'opposent sur les logiques mêmes qui président à l'organisation du trafic d'héroïne. Stringer Bell le fait à partir de l'application des règles de la macroéconomie qu'il apprend en formation continue au Baltimore City Community College. La qualité du produit devient pour lui l'enjeu des flux, et de l'efficacité du trafic, tandis qu'Avon Barksdale défend une ancienne conception liée à sa souveraineté sur un territoire déterminé. Ce sont donc deux visions de la société, et du « gouvernement » des hommes de main et des consommateurs, qui sont proposées dans ce débat fratricide qui s'ancre à la fois dans la communauté expérientielle des deux personnages (et de la série) et dans des scènes furtives où leur distance se marque. La transversalisation de la figure de l'homo oeconomicus à des secteurs d'activités considérés habituellement comme marginaux permet à chacun d'accéder à l'espace commun de la série, à partir de ses ressources propres. Le lieu des trafiquants n'est donc plus simplement celui de la marge et des secteurs défavorisés, mais devient celui de l'organisation capitaliste même. Les séries télévisées récitent donc des effets inédits du réel. Elles alimentent la contradiction, en mettant en scène d'un côté le modèle de la famille équilibrée avant la surve- nue d'une rupture biographique (perte d'emploi, accident, annonce de maladie), et de l'autre l'irruption de la déviance, portée par des personnages d'apparence populaire, mais qui conservent une part de facticité liée à leur icônisation.

La figure des " inadaptés », c'est-à-dire les classes, les groupes sociaux ou les professions habituellement délaissés par la fiction, traverse une partie des séries étudiées. L'opération de tressage de leurs caractéristiques réinvente la représentation de ces figures, et détaille l'écart entre leurs pratiques et attitudes et celles du monde familial idéal. Alors que le système visuel les a longtemps rendues «invisibles », elles acquièrent un droit à l'image : la représentation de la différence et de l'hétérogénéité contribue à l'écroulement de la représentation habituelle, altérant en partie les modèles ataviques que la société maintenait à travers les séries (et l'image en général). Les prisonniers d' $O z$ ou les drogués et les dockers de The Wire introduisent des ruptures fortes et proposent un décryptage alternatif du monde social. Ce dernier est désormais vu à travers leur perception. Dans The Wire, l'invitation régulière du personnage de Bubbles, toxicomane et indicateur de la police, à rendre des comptes, est un travail de citation qui consiste à rendre compréhensible cette incarnation de la figure de l'inadapté. Ce personnage reste cependant toujours inaccessible à lui-même, et scénaristiquement opaque. À travers ses séances de désintoxication, Bubbles retrouve son prénom, Reginald, mais ne parvient jamais à achever son traitement et à obtenir un nom de famille qui le rattacherait au groupe, même lorsqu'un journaliste en fait le sujet central de son reportage sur les sans-abri à la fin de la dernière saison. 
Sous les traits des détectives (The Wire, True Detective), des administrateurs de prison $(\mathrm{Oz})$, des militaires (Homeland), ou des agents antiterroristes $(24 h)$, la figure des forces de l'ordre est la dernière icône constitutive du présent des séries contemporaines. Cet ordre s'incarne visiblement dans une série comme $O z$, « série panoptique » qui se propose de figurer une société totalement disciplinaire, au sens de Foucault. $\mathrm{Oz}$ présente l'utopie carcérale d'une transparence absolue, et au fond, transforme ses spectateurs en représentants du maintien de l'ordre (la caméra entre dans les cellules), dont la figure est alors extraite de la fiction au profit de la vision même. Dans $O z$, Les Experts, 24 chrono, The Shield ou The Wire, c'est en grande partie à travers l'œil des forces de l'ordre et celui de la surveillance que le spectateur voit les relations sociales, économiques et politiques susceptibles d'être renouvelées ou inventées. De façon performative, les forces de l'ordre organisent le rassemblement des communautés sérielles, en les saisissant du point de vue d'un narrateur empathique. Mais cette communauté n'est pas nécessairement le pendant d'une réalité omnipotente sur la fiction. Les pratiques, les ressources comme les principes des forces de l'ordre montrent souvent des ajustements, des nouveautés et des résistances. On retrouve alors ici le premier ordre de la négation de l'utopie, tel que proposé par Rancière : l'ordre de la domination, qui assigne les corps dans des lieux déterminés, c'est ce que Rancière avait appelé « l'ordre policier », le partage entre ceux qui obéissent et ceux qui commandent ${ }^{14}$.

Les séries télévisées sont au final un prisme de temps qui réfléchit mais surtout réfracte et déforme ses propres contenus et ses formes, par la représentation verticale des corps « légitimes et illégitimes », la prolifération des symboles dans le présent de la fiction, et la condensation des aspects classiques comme innovants des récits. Le politique est replacé dans l'invisibilité du quotidien, soit l'échange normal et courant. Il ne se perçoit pas, à première vue, parce que l'échange a l'apparence routinière et conventionnelle de l'habitude. Il se trouve pourtant dans la tension constante entre les cadres temporels en vigueur et les tendances à les déborder. Ainsi, les séries contemporaines cherchent des espaces autonomes qui impliquent de nouveaux sens et figures.

\section{Les propositions utopiques de la fiction}

Derrière le réalisme affiché des séries contemporaines, il est possible de découvrir un retour assumé du fictionnel. Ce retour passe par la proposition d'univers fantastiques ( $X$-Files, Lost, Once upon a time, Game of Thrones, Battlestar Galactica, etc.), par la plongée dans des mondes socio-professionnels méconnus «plus vrais que nature » et prenant des libertés avec le réel (Urgences $^{15}$, À la Maison-Blanche), ou encore par des mécanismes narratifs permettant au récit de se montrer comme tel ${ }^{16}$. Mais cela passe aussi, pour ce qui nous intéresse, par la mise en scène d'un présent fictionnel de la série qui s'apparente à une historiographie d'autant de futurs possibles. Ainsi, ces séries fonctionnent moins comme un « mode d'emploi » du monde réel que comme un écart avec ce qu'il est. De la zone expérimentale d'Hamsterdam, dans The Wire, où le deal est toléré ${ }^{17}$, à tous les scénarios fonctionnant purement sur le mode du « et si » d'À la Maison-Blanche (« et si un essai nucléaire réalisé par un État inconnu était repéré par les 
satellites ", " et si le speaker de la Chambre tentait d'humilier le président », etc.), et de The Leftovers ( $«$ et si $2 \%$ de la population mondiale disparaissaient du jour au lendemain sans aucune explication »), les exemples abondent du choix d'un temps qui se veut anticipation. Temps qui imagine les manières non plus seulement de surmonter une situation problématique (ce que faisaient les séries classiques), mais de refaire société.

Dans le temps de The Leftovers, les disparitions massives et inexpliquées défont irrémédiablement les liens sociaux et familiaux, rendant impossible tout retour à la normale, et provoquant un immense trouble sociétal et individuel, qui consume tous les protagonistes. À l'inverse de The Leftovers, la série Les 4400 imaginait les enjeux sociétaux du retour soudain de 4400 personnes portées disparues depuis des décennies. Dans Terra Nova, l'extrême pollution sur une terre du futur conduit divers habitants sélectionnés à aller repeupler le monde du passé, du temps des dinosaures, et à tout réinventer dans cet environnement hostile, et ce nouvel état de nature, dont Lost avait aussi fait son point de départ. Le problème de cette solution est qu'elle escamote toute idée d'une communauté au présent, puisqu'elle la fait partir du futur pour aller vers le passé. La série suspend l'idée que notre présent est le passé de la narration, en la plaçant dans un avenir proche, pour immédiatement proposer un futur possible de l'humanité situé dans le passé. L'irréalisme de cette solution pour refaire communauté, et l'aporie intellectuelle qu'elle contient (pour se refonder, le groupe doit se projeter, mais vers le passé), conjugués à des faiblesses de scénario, expliquent sans doute que cette série n'ait tenu qu'une saison, malgré la présence de Steven Spielberg à la production.

On voit bien avec ces exemples combien la question d'un temps présent alternatif est centrale scénaristiquement. Toutes ces séries exposent et reconduisent donc un trouble dans le monde social et un trouble des êtres, qui fonde le récit, sans pour autant chercher à en proposer une résolution univoque. Rien de « réaliste» ici, au sens documentaire du terme, mais seulement du plausible, de l'hypothétique, et du fictionnel. Même les séries à visée réaliste (The Wire, Les Experts, etc.) ne sont que des narrations scénarisées, mettant en scène des situations et des personnages qui n'ont jamais existé et n'existeront jamais, car elles parlent d'un autre temps, et depuis un autre temps, celui de la fiction et de ses codes.

Dans ces futurs possibles, ce n'est plus l'identification à un ou à plusieurs personnages qui est recherchée, car les éléments qui la permettraient sont absents ou suspendus. Les protagonistes des séries contemporaines n'ont en effet plus nécessairement d'attributs héroïques, et, comme cela a pu être remarqué, ils sont protéiformes et changeants, névrosés aussi $1^{18}$. Leur « complexité » témoigne de leur humanité, et en fait non des hommes sans qualité, mais des individus se débattant dans un univers familier au nôtre, mais irrémédiablement décalé. La quête poursuivie par certains protagonistes peut éventuellement susciter l'adhésion (empêcher un attentat, sauver sa famille, etc.), mais les moyens qu'ils emploient, comme leur dépravation morale, rendent l'identification difficile : Jack Bauer dans 24 chrono, Tony Soprano, les policiers ripoux 
de The Shield, les dealers de The Wire, le tueur en série justicier de Dexter, etc. Et, au passage, il est intéressant de noter que des personnages relevant habituellement d'univers opposés, voire en conflit, se retrouvent désormais compris dans une même réprobation morale. La fiction des séries contemporaines permet donc de supposer un discours autre qui fait surgir des pratiques encore virtuelles, des situations non-advenues, qui poussent à imaginer et penser d'autres manières de faire et de montrer que celles du temps passé ou des présents pluriels.

La suspension de l'identification apparaît ainsi comme la condition du triomphe du temps alternatif proposé, car ce qui se joue est moins la définition même du personnage que ce qu'il va pouvoir faire dans le présent qui est le sien. Pour s'arrêter sur un cas donné, il nous semble que True Blood, au moins dans ses trois premières saisons, propose à ses héros d'exister dans une société absolument permissive. True Blood joue bien sûr sur son espace propre, celui du Sud américain, du bayou, loin des séries urbaines. Mais la série s'inscrit d'abord dans le temps de la nuit, nuit permanente, où les lois sont comme suspendues, où les êtres changent d'aspect, révèlent leur vraie nature (mutante, violente, animale, vampire), et paraissent détachés des contraintes diurnes, libres alors de n'être plus préoccupés que par leurs problèmes d'identité. Ensuite, la série retient de l'idée de permissivité, c'est-à-dire, si l'on s'appuie par exemple sur ce qu'ont pu en dire certains travaux à la suite de Norbert Elias ${ }^{19}$, la libération des pulsions. Le temps de True Blood met en scène ce à quoi ressemblerait une société où l'autocontrôle ne serait plus qu'épisodique. Et précisément, c'est le fait que les personnages cèdent à leurs envies qui est le moteur de l'action. Car il faut toujours rattraper une action qui n'aurait pas dû se produire (meurtre, prise de drogues, etc.). La seule tyrannie est celle des pulsions, car précisément la série fonctionne sur l'abandon de soi, le principe de plaisir et le refus des contraintes, comme conditions d'une société libérée. Ainsi, la série contient plusieurs séquences où la consommation de drogue (ici une drogue, le $\mathrm{V}$, tirée justement des vampires, littéralement personnages enchanteurs) est magnifiée, paradisiaque, et qui plus est partagée à plusieurs, car la drogue a cette propriété. De même, la deuxième saison est ponctuée de longues séquences d'orgies et de transes, liées à l'arrivée d'un personnage qui prend possession des esprits.

Abandon aussi du corps à ses capacités de transformation : nombre de personnages qui ont la faculté de pouvoir changer de forme et de s'animaliser expérimentent souvent la métamorphose. Jusqu'à en faire un art de vivre, si l'on pense par exemple au personnage de Sam, qui la nuit se change en animal, seul ou avec ses congénères, pour chercher une communion avec la nature, pour renouer avec une puissance pastorale, que le temps diurne interdit. Comme dans Terra Nova ou Lost, c'est d'un véritable « retour à la nature » qu'il s'agit, physiologique cette fois, visible dans la nudité fréquente des protagonistes, soit parce qu'ils sont nus lorsqu'ils retrouvent forme humaine, soit parce que la série les saisit dans leur activité sexuelle. Toutes les saisons sont d'ailleurs traversées de fortes tensions sexuelles entre les personnages, et de scènes de sexe explicites, homosexuelles ou hétérosexuelles, entre humains seulement, ou entre humains, vampires, et autres créatures, dans un triomphe débridé des corps qui 
n'empêche cependant pas le désir trouble et la présence récurrente du sang (les vampires mêlent sexe et morsure). Enfin, la série n'omet pas de montrer que le relâchement des contrôles conduit aussi à la violence, et son usage est récurrent, sous différentes formes (crimes de haine à l'égard des vampires, expéditions punitives, crimes perpétrés par les vampires eux-mêmes, etc.) sans que les personnages s'interrogent plus avant sur son utilisation.

True Blood met donc en scène un temps autre où tout est permis, où les figures de la loi sont veules et faibles, et où les êtres consentent aux pulsions sans chercher à les refréner. Le déplacement de l'action dans l'espace mystérieux du sud des États-Unis apparaît ainsi comme la condition de création d'un temps fictionnel "libertaire ", hédoniste, où les comportements sexuels et addictifs ne font pas l'objet d'un saisissement moral ou policier, mais traversent naturellement les personnages comme des états normaux du corps. L'utopie d'une société débarrassée de ses corsets puritains fonde le propos de True Blood, et permet de dépeindre des personnages dont l'accomplissement se jouera ailleurs (amour, triomphe politique) que dans la transgression des interdits sociaux, ici tenue pour acquise.

Les analyses et interprétations qui précèdent ont permis de prendre de la distance avec la tendance à construire les séries télévisées contemporaines comme un phénomène $a d h o c$, qui permettrait de mettre en lumière des phénomènes sociaux « de la vie réelle ", ou même de proposer une lecture politique de ces mêmes phénomènes (ce qui est trop souvent fait avec The Wire). L'engagement politique des séries s'établit à travers la mise en scène d'un temps social et politique justement alternatif. L'espace commun à la série et à la réalité est donc celui de la dispute pour le sens du temps, que ce soit par leur lente temporalité qui émerge dans l'opération de mise en série ; par la proposition d'une pluralité de présents vraisemblables ; ou dans l'anticipation fictionnelle. Les séries contemporaines inscrivent donc les (re)-configurations d'une idée de réalité dans la visualité du contexte de leur diffusion. Telle une pédagogie de l'actualité, leur participation au système d'évidences sensibles limite l'accès au commun en répartissant le temps et l'espace réservés à chacun dans la communautée ${ }^{20}$. Leur rapport au temps réel participe à la tâche du politique, c'est-à-dire la fondation symbolique du pouvoir et sa permanente justification, et donc son remplissage par l'imagination visuelle avec sa performativité, sa dramaturgie et son spectacle.

Pour autant, ces représentations utopiques de sociétés encore à penser n'installent pas de dissensus, au sens de Jacques Rancière, puisqu'elles restent des propositions et des anticipations à l'état de mirage représentationnel et non dans l'art lui-même, ce que Rancière nommerait le sensible. Comme le note justement Philippe Mangeot, « [c]es œuvres au long cours qui se produisent au fur et à mesure de leur visionnage sont des ateliers à ciel ouvert, riches en repentirs impossibles à masquer, en résolutions locales qui ouvrent de nouveaux problèmes ${ }^{21}$. Pour reprendre l'articulation de l'écriture de l'histoire chère à Michel de Certeau, les énonciations alternatives des séries contemporaines lient des variations du discours (au sens foucaldien d'un « nous » conventionnel) dans des récits pluriels pour réaliser la fiction d'une pluralité des possibles, faits de normes 
partagées comme de contre-conduites mises en scène. Les séries deviennent donc des légendes, au double sens du terme : des récits, dont la part de réalisme historique est déformée ; un repère de lecture de la topographie des possibilités et des impossibilités. Elles offrent, à partir d'une parole propre, une forme de critique du pouvoir et des institutions, ou en tout cas une ouverture des possibles, qui revient malgré tout sans cesse au principe ordonnateur : le temps devenu prémonition.

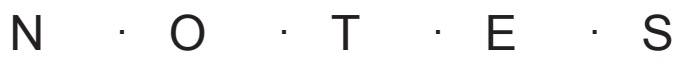

1. Voir par exemple, Marie-Hélène Bacqué, Amélie Flamand, Caroline Glorion, Anne-Marie Paquet-Deyris et Julien Talpin, « The Wire, visages du ghetto : entre fiction et sciences sociales », les 26 et 27 octobre 2012 ; ces journées viennent ponctuer le travail du séminaire " The Wire, a fiction in the ghetto », qui a eu lieu durant l'année universitaire 2011-2012 à l'Université Paris X - Nanterre. Cf. M.-H. Bacqué, A. Flamand, A.-M. Paquet-Deyris, J. Talpin (dir.), The Wire. L'Amérique sur écoute, Paris, La Découverte, 2014.

2. Joseph Belletante, «Contribution des séries américaines à la formation du jugement politique », thèse de doctorat en science politique dirigée par Jean-Louis Marie, Université Lumière - Lyon II, 2008 ; publiée sous le titre Séries et politique. Quand la fiction contribue à l'opinion (Paris, L'Harmattan, coll. « Champs visuels », 2011).

3. Serge Daney, «Il y a séries et séries » in Le salaire du zappeur, Paris, Libération-Ramsay, coll. « Poche cinéma », 1987, pp. 111-115. Sur Columbo, on dispose désormais de l'ouvrage de Lilian Mathieu, Columbo : la lutte des classes ce soir à la télé, Paris, Textuel, 2013. 4. Jusqu'à en être complètement détaché aujourd'hui dans les modes de consommation. La série peut être achetée en dvd ou téléchargée, et regardée sur un ordinateur, un vidéoprojecteur, etc., sans dépendance aux programmes de télévision, et à l'objet téléviseur. 5. À noter qu'une troisième saison regroupant neuf épisodes écrits, produits et dirigés par les créateurs originaux est en projet sur la chaîne américaine Showtime, pour une diffusion en 2016, à l'occasion des vingt-cinq ans de son arrêt sur ABC. Pour une lecture ésotérique de cette série, voir Pacôme Thiellement, $L a$ main gauche de David Lynch. Twin Peaks et la fin de la télévision, Paris, PUF, coll. « Travaux pratiques », 
2010.

6. Michel Kokoreff, "Sérialité et répétition : l'esthétique télévisuelle en question », Quaderni, n 9 , hiver 1989/1990, p. 21.

7. Nous pensons également ici aux séries qui prennent le même format et qui sont diffusées par des networks tels que Showtime ou ABC, par Netflix aussi, aux États-Unis, la BBC en Angleterre ou Canal + en France.

8. On retrouve ici deux des trois ordres de la négation de l'utopie selon Jacques Rancière. Il écrit que l'ordre politique « vient interrompre l'ordre policier en imposant un compte surnuméraire des incomptés », et en faisant qu'ils sont entendus. Le projet utopique va « redonner consistance à la communauté in-consistante de la politique », en proposant un ordre où les corps soient à leur vraie place. Voir Jacques Rancière, $L a$ Mésentente, Paris, Galilée, 1995, pp. 74-76.

9. Gilles Deleuze, Cinéma, I. L'image-mouvement, Paris, Éditions de Minuit, 1983.

10. Pour Jean-Marie Samocki, le rythme particulier de The Wire tient au fait que les actions importantes sont systématiquement accélérées ou différées. J.-M. Samocki, « Sans appel », in Emmanuel Burdeau, Nicolas Vieillescazes (dir.), The Wire. Reconstitution collective, Paris, Les prairies ordinaires, 2011, p. 37. 11. David Buxton, Les séries télévisées. Forme, idéologie et mode de production, Paris, L'Harmattan, 2010, p. 16.

12. Michel de Certeau, L'écriture de l'histoire, Paris, Gallimard, 1975.

13. Carlo Ginzburg, «Signes, traces, pistes. Racines d'un paradigme de l'indice», Le débat, 6 novembre 1980, pp. 3-44.

14. Jacques Rancière, La Mésentente, op. cit., p. 73.

15. Jean Peneff montre bien comment une série comme Urgences joue simultanément sur un réalisme technique et professionnel, tout en forçant des traits et en en passant d'autres sous silence. Jean Peneff, « La face cachée d'Urgences, le feuilleton de la télévision », Genèses, n³0, 1998.

16. C'est le cas avec le recours à une voix-off émanant d'un narrateur omniscient. Héloïse Pourtier-Tillinac, « La fin du réalisme dans les séries télévisées. La narration à portée généralisante, un tournant télévisuel ? », Réseaux, «Les séries télévisées », n 165 , février-mars 2011.

17. D'autres exemples propres à ce drama pourraient être mobilisés : le déroulement de la saison 5 autour d'un vrai-faux tueur en série mis en scène par le détective Jimmy McNulty, ou encore le choix de Wee-Bey Brice, trafiquant incarcéré qui, dans la saison 4, accepte de confier son fils au major Colvin (en lui attribuant son tutorat contre l'avis de sa propre épouse) pour lui ouvrir d'autres possibilités que celles de devenir dealer.

18. Joseph Belletante, «Le désert des héros : récits de vies solitaires dans les séries américaines de fiction », Conserveries mémorielles [en ligne], nº 7, 2010, disponible sur http://cm.revues.org/439.

19. Stephen Mennell, "Decivilising Process: Theoretical Significance and Some Lines of Research », International Sociology, vol. 5, n², juin1990.

20. On trouve ici la définition de « topographique du possible » que donne Jacques Rancière. Voir J. Rancière, Le spectateur émancipé, Paris, La Fabrique, 2008, p. 55.

21. Philippe Mangeot, "Saison 4. Genèses », in Emmanuel Burdeau, Nicolas Vieillescazes (dir.), The Wire, op. cit., p. 92. 


\title{
$\mathrm{R} \cdot \hat{E} \cdot \mathrm{S} \cdot \mathrm{U} \cdot \mathrm{M} \cdot \mathrm{E}$ expose the gap between the series and the actual world.
}

À partir du constat d'un saut qualitatif dans la fabrication des séries télévisées contemporaines, ce texte questionne l'autonomisation $\mathrm{du}$ matériau filmique des drama, à travers l'organisation de leur temporalité. Ces séries offrent en effet une réflexion politique qui porte sur le sens du temps collectif. L'hypothèse est que le temps mis en scène dans les séries est celui d'une politique alternative, un temps autre, fait d'anticipations et de propositions capables de refonder la communauté. À partir de trois mouvements : l'opération de mise en série qui joue sur une narration complexe et lente ; la vraisemblance d'une pluralité de présents, et de figures nouvelles capables de les incarner; et un certain nombre de propositions utopiques, qui se conjuguent au futur dans l'écart entre les séries et le monde réel.

\begin{abstract}
Based on the observation of a qualitative leap in the production of contemporary television series, this article questions the increasing autonomy of dramas' film material, through the organization of their temporality. These TV shows offer a political analysis on the meaning of collective time. The assumption is that time as it is staged in TV shows is a time of a political alternative; which is composed of anticipations and proposals able to rebuild the community. This alternate time originates in three movements: the serialization operation that builds a complex and slow narration; the likelihood of a plurality of present times and new characters able to embody them; and utopian proposals, which
\end{abstract}


